\title{
Benchmark Quantum Mechanical Calculations of Vibrationally Resolved Cross Sections and Rate Constants on ab initio Potential Energy Surfaces for the F+HD Reaction: Comparisons with Experiments \\ Dario De Fazio \\ Istituto di Struttura della materia - C.N.R., 00016 Roma, Italy \\ Simonetta Cavalli, Vincenzo Aquilanti \\ Dipartimento di Chimica, Biologia e Biotecnologie - Università di Perugia, 06123 Perugia, Italy
}

May 16, 2016

\section{Supporting Information}

Table S1. Comparison of the calculated rate constants (in $\mathrm{cm}^{3}$ molecule ${ }^{-1} \mathrm{~s}^{-1} \times 10^{-13}$ ) for the HF formation on the LWAL, CSZ, FXZ and SW PESs as a function of temperature. 


\begin{tabular}{|c|c|c|c|c|c|c|c|}
\hline \multirow[t]{2}{*}{$\mathrm{T}(\mathrm{K})$} & \multirow{2}{*}{$\begin{array}{c}\text { LWAL } \\
\text { This Work }\end{array}$} & \multirow{2}{*}{$\begin{array}{c}\text { CSZ } \\
\text { This Work }\end{array}$} & \multicolumn{2}{|l|}{ FXZ } & \multicolumn{3}{|c|}{ SW } \\
\hline & & & This Work & Ref. $^{1}$ & This Work & Ref. $^{2}$ & Ref. $^{3}$ \\
\hline 10 & .630 & .837 & .470 & & 2.51 & & \\
\hline 20 & .849 & .944 & .524 & & 2.36 & & \\
\hline 40 & 3.68 & 3.64 & 2.19 & & 4.99 & & \\
\hline 60 & 8.52 & 8.54 & 5.91 & & 11.7 & & \\
\hline 80 & 13.0 & 13.2 & 9.83 & & 19.8 & & \\
\hline 100 & 17.4 & 17.7 & 13.7 & 13.7 & 27.9 & 28.7 & 27.1 \\
\hline 120 & 22.2 & 22.6 & 17.9 & 17.9 & 35.8 & 36.4 & 35.4 \\
\hline 140 & 27.8 & 28.3 & 22.8 & 22.8 & 44.0 & 44.3 & 43.9 \\
\hline 160 & 34.5 & 35.1 & 28.7 & 28.7 & 52.7 & 52.6 & 52.9 \\
\hline 180 & 42.1 & 42.9 & 35.5 & 35.5 & 61.8 & 61.4 & 62.2 \\
\hline 200 & 50.6 & 51.5 & 43.1 & 43.1 & 71.4 & 70.7 & 72.0 \\
\hline 220 & 59.7 & 60.7 & 51.5 & 51.4 & 81.5 & 80.3 & 82.2 \\
\hline 240 & 69.2 & 70.4 & 60.4 & 60.2 & 91.8 & 90.1 & 92.7 \\
\hline 260 & 79.1 & 80.5 & 69.8 & 69.4 & 102 & 100 & 103 \\
\hline 280 & 89.2 & 90.8 & 79.4 & 78.8 & 113 & 110 & 114 \\
\hline 300 & 99.5 & 101 & 89.4 & 88.3 & 124 & 120 & 125 \\
\hline 320 & 110 & 112 & & & 134 & & \\
\hline 340 & 120 & 122 & & & 145 & & \\
\hline 350 & 125 & 127 & & & 150 & & 152 \\
\hline
\end{tabular}


Table S2. Comparison of the calculated rate constants (in $\mathrm{cm}^{3}$ molecule ${ }^{-1} \mathrm{~s}^{-1} \times 10^{-13}$ ) for the DF formation on the LWAL, CSZ, FXZ and SW PESs as a function of temperature.

\begin{tabular}{|c|c|c|c|c|c|c|c|}
\hline \multirow[t]{2}{*}{$\mathrm{T}(\mathrm{K})$} & \multirow{2}{*}{$\begin{array}{c}\text { LWAL } \\
\text { This Work }\end{array}$} & \multirow{2}{*}{$\begin{array}{c}\text { CSZ } \\
\text { This Work }\end{array}$} & \multicolumn{2}{|c|}{ FXZ } & \multicolumn{3}{|c|}{ SW } \\
\hline & & & This Work & Ref. $^{1}$ & This Work & Ref. $^{2}$ & Ref. $^{3}$ \\
\hline 10 & .067 & .099 & .065 & & .469 & & \\
\hline 20 & .077 & .099 & .066 & & .423 & & \\
\hline 40 & .155 & .177 & .122 & & .600 & & \\
\hline 60 & .391 & .418 & .292 & & 1.12 & & \\
\hline 80 & 1.00 & 1.03 & .735 & & 2.28 & & \\
\hline 100 & 2.24 & 2.28 & 1.67 & 1.67 & 4.39 & 4.3 & 4.36 \\
\hline 120 & 4.31 & 4.36 & 3.29 & 3.29 & 7.63 & 7.6 & 7.61 \\
\hline 140 & 7.30 & 7.36 & 5.71 & 5.71 & 12.0 & 11.9 & 11.9 \\
\hline 160 & 11.2 & 11.3 & 8.99 & 8.99 & 17.4 & 17.3 & 17.4 \\
\hline 180 & 16.0 & 16.1 & 13.1 & 13.0 & 23.7 & 23.6 & 23.7 \\
\hline 200 & 21.5 & 21.6 & 17.9 & 17.8 & 30.8 & 30.6 & 30.7 \\
\hline 220 & 27.7 & 27.8 & 23.4 & 23.3 & 38.5 & 38.2 & 38.3 \\
\hline 240 & 34.4 & 34.5 & 29.4 & 29.2 & 46.6 & 46.3 & 46.3 \\
\hline 260 & 41.5 & 41.6 & 35.9 & 35.5 & 55.0 & 54.6 & 54.6 \\
\hline 280 & 48.9 & 49.0 & 42.7 & 42.1 & 63.5 & 63.1 & 63.1 \\
\hline 300 & 56.5 & 56.5 & 49.8 & 48.9 & 72.2 & 71.7 & 71.7 \\
\hline 320 & 64.1 & 64.2 & & & 80.6 & & \\
\hline 340 & 71.8 & 71.9 & & & 89.4 & & \\
\hline 350 & 75.7 & 75.7 & & & 93.6 & & 93.4 \\
\hline
\end{tabular}

\section{References}

[1] De Fazio, D.; Lucas, J. M.; Aquilanti , V.; Cavalli, S. Exploring the accuracy level of new potential energy surfaces for the F + HD reactions: from exact quantum rate constants to the state-to-state reaction dynamics. Phys. Chem. Chem. Phys. 2011, 13, 8571-8582. 
[2] De Fazio, D.; Aquilanti, V.; Cavalli, S.; Aguilar, A.; Lucas, J. M. Exact quantum calculations of the kinetic isotope effect: cross sections and rate constants for the $\mathrm{F}+\mathrm{HD}$ reaction and role of tunnelling. J. Chem. Phys. 2006,125, 133109.

[3] Zhang, D.H.; Lee, S.-Y.; Baer, M. Quantum mechanical integral cross sections and rate constants for the F+HD reactions. J. Chem. Phys. 2000, 112, 9802-9809. 